J. Dairy Sci. 92:382-391
doi:10.3168/jds.2008-1310
(c) American Dairy Science Association, 2009.

\title{
Economic evaluation of genomic breeding programs
}

\author{
S. König,$^{* 1}$ H. Simianer, ${ }^{*}$ and A. Willam† \\ *Institute of Animal Breeding and Genetics, University of Göttingen, 37075 Göttingen, Germany \\ †Division of Livestock Sciences, BOKU-University of Natural Resources and Applied Life Sciences, 1180 Vienna, Austria
}

\begin{abstract}
The objective of this study was to compare a conventional dairy cattle breeding program characterized by a progeny testing scheme with different scenarios of genomic breeding programs. The ultimate economic evaluation criterion was discounted profit reflecting discounted returns minus discounted costs per cow in a balanced breeding goal of production and functionality. A deterministic approach mainly based on the gene flow method and selection index calculations was used to model a conventional progeny testing program and different scenarios of genomic breeding programs. As a novel idea, the modeling of the genomic breeding program accounted for the proportion of farmers waiting for daughter records of genotyped young bulls before using them for artificial insemination. Technical and biological coefficients for modeling were chosen to correspond to a German breeding organization. The conventional breeding program for 50 test bulls per year within a population of 100,000 cows served as a base scenario. Scenarios of genomic breeding programs considered the variation of costs for genotyping, selection intensity of cow sires, proportion of farmers waiting for daughter records of genotyped young bulls, and different accuracies of genomic indices for bulls and cows. Given that the accuracies of genomic indices are greater than 0.70 , a distinct economic advantage was found for all scenarios of genomic breeding programs up to factor 2.59, mainly due to the reduction in generation intervals. Costs for genotyping were negligible when focusing on a population-wide perspective and considering additional costs for herdbook registration, milk recording, or keeping of bulls, especially if there is no need for yearly recalculation of effects of single nucleotide polymorphisms. Genomic breeding programs generated a higher discounted profit than a conventional progeny testing program for all scenarios where at least $20 \%$ of the inseminations were done by genotyped young bulls without daughter records. Evalua-
\end{abstract}

Received April 29, 2008.

Accepted September 11, 2008.

${ }^{1}$ Corresponding author: skoenig2@gwdg.de tion of levels of annual genetic gain for individual traits revealed the same potential for low heritable traits $\left(\mathrm{h}^{2}\right.$ $=0.05)$ compared with moderate heritable traits $\left(\mathrm{h}^{2}=\right.$ 0.30 ), preconditioning highly accurate genomic indices of 0.90. The final economic success of genomic breeding programs strongly depends on the complete abdication of any forms of progeny testing to reduce costs and generation intervals, but such a strategy implies the willingness of the participating milk producers.

Key words: genomic selection, breeding program, economics, deterministic approach

\section{INTRODUCTION}

The implementation of AI enabled the successful realization of progeny testing (PT) programs for dairy cattle as suggested by Henderson (1964). As a major disadvantage, PT implies generation intervals longer than $5 \mathrm{yr}$ for all paths of selection (König et al., 2007). A second concern is related to the costs of the breeding program. Assuming a waiting period of $4.5 \mathrm{yr}$ of young test bulls and feeding and keeping costs of 5 euro per bull per day, $32 \%$ of the costs for PT are related to the unproductive waiting period of bulls from finishing inseminations until breeding value estimation (Leisen, 1999). Several ideas arose to reduce generation intervals or to improve existing PT programs, such as multiple ovulation and embryo transfer breeding schemes (Nicholas and Smith, 1983) or marker-assisted selection (e.g., Mackinnon and George, 1998), but practical results lagged behind theoretical expectations.

Genomic selection (GS) provides the opportunity for substantial modifications to both genetic evaluation system and breeding program design. Genomic selection is possible due to a multitude of so-called SNP, distributed fairly evenly over the whole genome, so that all genes are expected to be in linkage disequilibrium with these markers (Meuwissen et al., 2001). The genomic estimated breeding value (GEBV) considers in its calculation all single SNP effects. Reliable GEBV for both sexes with accuracies greater than 0.70 can be calculated at an early stage of an animal's life (e.g., even for embryos). This implies a shift from BLUP animal models including pedigree information toward 


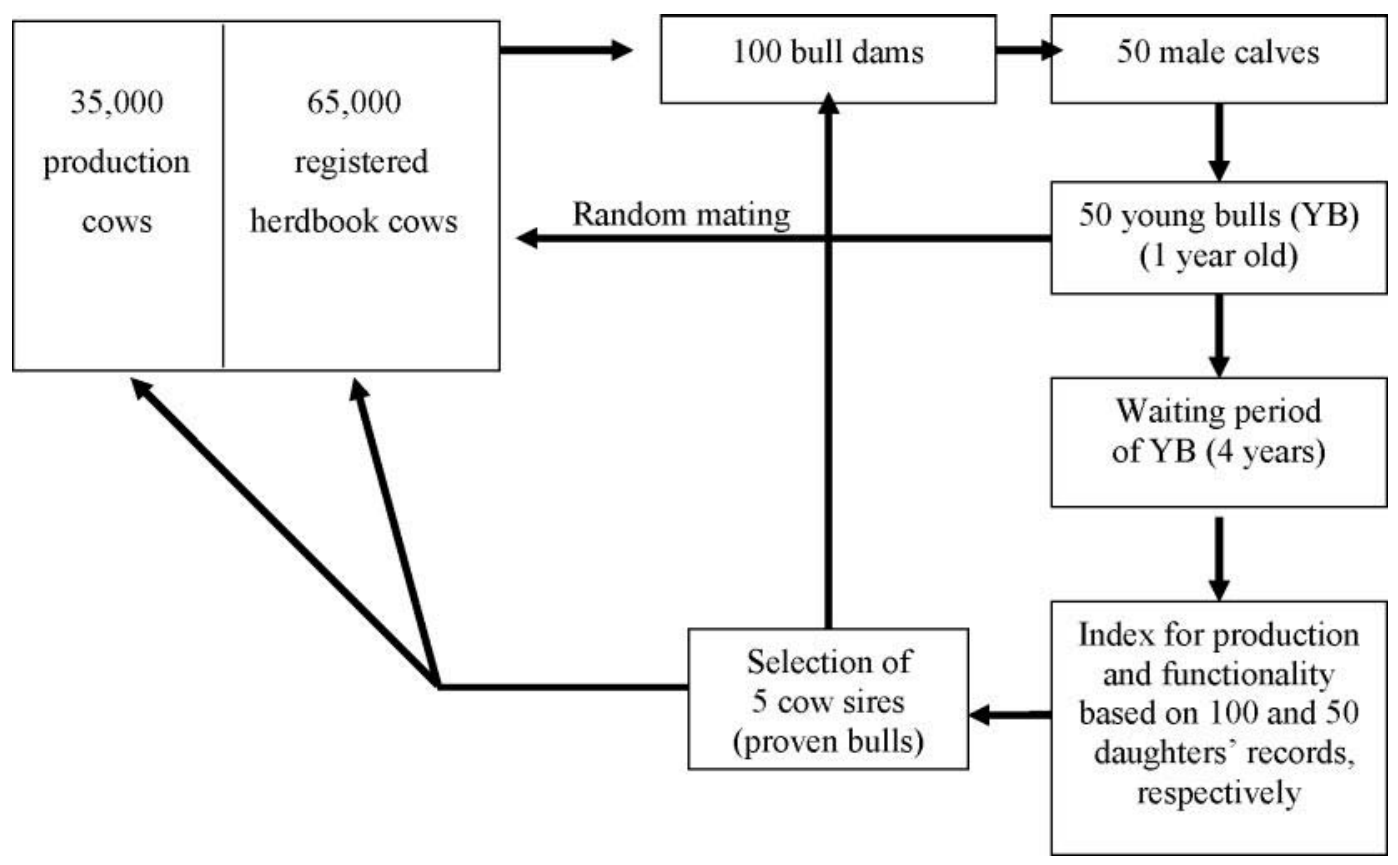

Figure 1. Conventional progeny testing program (CPTP) for the final selection of 5 cow sires per year.

SNP-based BLUP and putting less weight on information provided by relatives. A variety of methods have been suggested for the estimation of genomic breeding values [e.g., BLUP (Kolbehdari et al., 2007), Bayesian methods (Meuwissen et al., 2001), or machine learning procedures (Long et al., 2007)].

The availability of high-density marker maps as used for GS will increase annual genetic gain and provide new tools for the management of inbreeding as shown by Dekkers (2007). Schaeffer (2006) assumed an accuracy of 0.75 for GEBV and applied the general formula by Rendel and Robertson (1950) for calculating annual genetic gain on 4 paths of selection of the Canadian Holstein population. Annual genetic gain was doubled due to a substantial reduction of generation intervals, and costs of proving bulls were reduced by $92 \%$ due to the elimination of PT. However, there is a substantial lack of scientific studies evaluating GS economically from a population-wide perspective (i.e., considering discounted fixed and variable breeding costs and returns, as well as biological and technical coefficients in the given investment period).

The objective of the present study was to evaluate how the design of the conventional PT program for Holstein dairy cattle in Germany can be modified toward a genomic breeding program to optimize annual monetary genetic gain and discounted profit for a variety of practical restrictions in various breeding scenarios.

\section{MATERIALS AND METHODS}

A conventional PT program (CPTP) for 50 young bulls per year and a genomic breeding program (GBP) of the same population size of 100,000 Holstein cows were modeled applying the computer program ZPLAN (Nitter et al., 2007). The main characteristics of the CPTP are depicted in Figure 1. One hundred bull dams are selected using EBV, phenotypic performances, and pedigree indices to produce 50 male calves for the AI center. Biotechnologies to increase the number of offspring per bull dam, such as embryo transfer, were not considered in all breeding scenarios. At the age of $1 \mathrm{yr}$, young bulls are randomly mated with registered herdbook cows to generate 100 daughter records of a production trait and 50 daughter records of a functional trait per bull for genetic evaluation. Final selection of 5 cow sires used for inseminations in the whole population is based on a combined breeding goal of production and functionality.

The general structure of the GBP for the selection of 5 cow sires per year is depicted in Figure 2. Identical to the CPTP, the active breeding stock includes 65,000 registered herdbook cows serving as potential bull dams. Implementation of GS needs genotyping an initial sample size of bulls to estimate SNP or haplotype effects. According to Schaeffer (2006), the estimates are based on 50 sires with 50 sons each, and each son has reliable EBV or daughter yield deviations based 
Table 1. Gene flow matrix for conventional progeny testing program (CPTP); arrows indicate the gene flow

\begin{tabular}{lccc} 
& \multicolumn{3}{c}{ Selection group } \\
\cline { 2 - 4 } Item & Proven bulls $(\mathrm{PB})$ & Herdbook cows $(\mathrm{HC})$ & Production cows (PC) \\
\hline $\mathrm{PB}$ & $1 . \mathrm{PB}^{1} \rightarrow \mathrm{PB}$ & $2 . \mathrm{HC}^{2} \rightarrow \mathrm{PB}$ & - \\
$\mathrm{HC}$ & $3 . \mathrm{YB}^{3} \rightarrow \mathrm{HC}$ & $5 . \mathrm{HC}^{4} \rightarrow \mathrm{HC}$ & - \\
& $4 . \mathrm{PB}^{1} \rightarrow \mathrm{HC}$ & - & $7 . \mathrm{PC} \rightarrow \mathrm{PC}$ \\
$\mathrm{PC}$ & $6 . \mathrm{PB} \rightarrow \mathrm{PC}$ & - & \\
\hline
\end{tabular}

${ }^{1} \mathrm{~PB}=$ proven bulls used as bull and dam sires.

${ }^{2} \mathrm{HC}=$ herdbook cows used as bull dams.

${ }^{3} \mathrm{YB}=$ young bulls used as cow sires.

${ }^{4} \mathrm{HC}=$ herdbook cows used as cow dams.

on more than 100 daughter records. The frequency for reestimating SNP effects is unknown. With new traits being defined and with improved methods to calculate GEBV, annual reestimation of SNP effects seems likely. A total of 200 elite heifers are preselected according to type traits and pedigree indices and genotyped at the age of 15 mo. One hundred bull dams with highest GEBV will be selected to generate 50 bull calves. Bull calves need to be genotyped, and the top 5 of them are used as cow and bull sires at the age of 12 mo for AI in the whole population of 100,000 cows for a time period of 3 years. This was taken as a base scenario with variations of some of these parameters also considered.

The ZPLAN is written in FORTRAN and allows a flexible modification of existing subroutines to model desired breeding scenarios as realistically as possible. Deterministic calculations, mainly based on the gene flow method (Hill, 1974) and selection index procedure, are used for the calculation of genetic and economic parameters after one round of selection. Selection index methodology was evaluated as a valid approach for estimation of selection response for different GS criteria in the study by Dekkers (2007), but ignores reduction in genetic variances due to selection termed as Bulmereffect (Falconer and Mackay, 1996). Consideration of such additional aspects would be possible when applying stochastic simulation, which requires more computing time than deterministic modeling. Defined selection groups and gene flow matrices for the CPTP and for GBP are given in Table 1 and Table 2, respectively.

Details for setting up a CPTP using ZPLAN were adopted from Willam et al. (2002), but were modified to the practical constraints of one German AI center. Essential biological and technical coefficients as well as breeding costs for modeling the CPTP in the present study are summarized in Table 3. A total of 50 young bulls were tested per year and $25 \%$ of recorded cows were mated with test bulls. This implies that the remaining $75 \%$ of cows will be mated with proven sires from this breeding program because gene flows from foreign proven sires or natural service sires were not considered in our model. Calculation of breeding costs considers activities of breeding organizations and AI centers, essentially herdbook registration per cow,

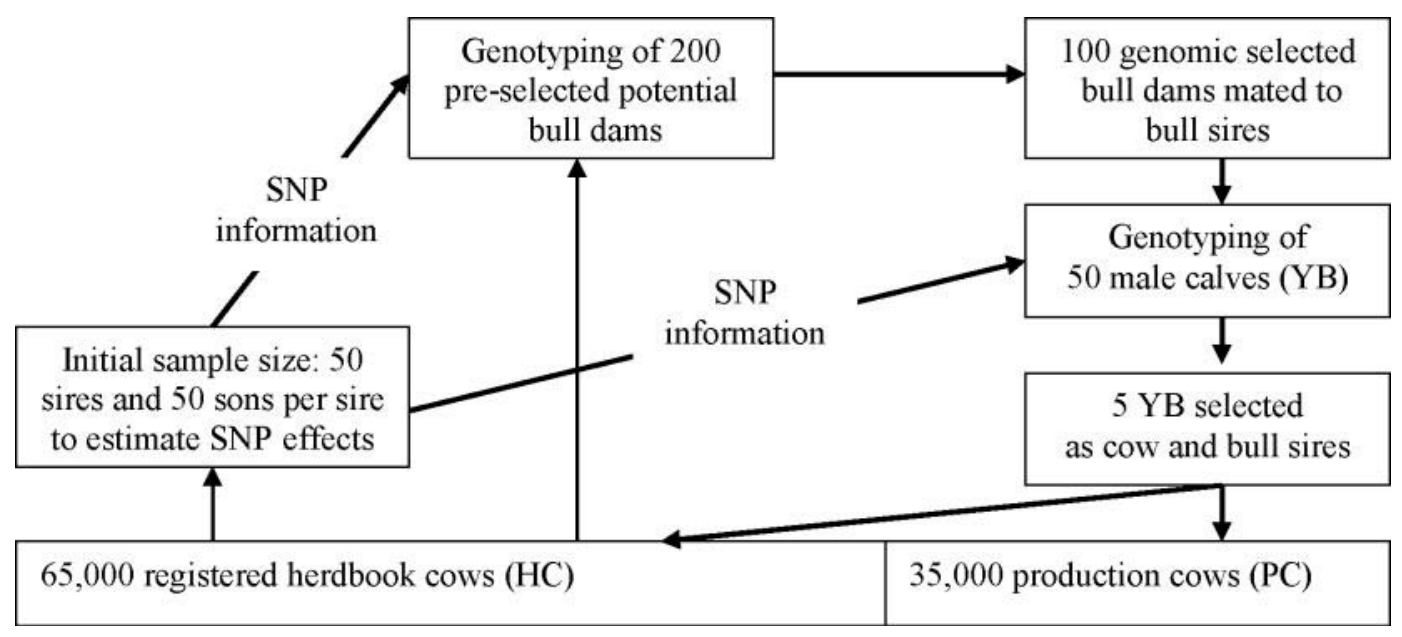

Figure 2. Possible genomic breeding program (GBP) for the final selection of 5 cow sires per year. 
Table 2. Gene flow matrix for genomic breeding program (GBP); arrows indicate the gene flow

\begin{tabular}{|c|c|c|c|}
\hline \multirow[b]{2}{*}{ Item } & \multicolumn{3}{|c|}{ Selection groups } \\
\hline & Young bulls (YB) & Herdbook cows (HC) & Production cows (PC) \\
\hline$\overline{\mathrm{YB}}$ & 1. $\mathrm{YB}^{1} \rightarrow \mathrm{YB}$ & 2. $\mathrm{HC}^{2} \rightarrow \mathrm{YB}$ & - \\
\hline $\mathrm{HC}$ & $\begin{array}{l}\text { 3. } \mathrm{YB}^{1} \rightarrow \mathrm{HC} \\
\text { 4. } \mathrm{YB}^{4} \rightarrow \mathrm{HC}\end{array}$ & 5. $\mathrm{HC}^{3} \rightarrow \mathrm{HC}$ & - \\
\hline $\mathrm{PC}$ & $\begin{array}{l}\text { 6. } \mathrm{YB} \rightarrow \mathrm{PC} \\
\text { 7. } \mathrm{YB}-\mathrm{D} \rightarrow \mathrm{PC}\end{array}$ & - & 8. $\mathrm{PC} \rightarrow \mathrm{PC}$ \\
\hline
\end{tabular}

financial contributions for milk recording, as well as fixed and variable costs for keeping bulls on station. For modeling the GBP, the additional cost component of 250 euro per animal for genotyping has to be taken into account. The interest rates for costs and return were 0.04 and 0.06 , respectively. An increased discount rate by 2 percentage points to evaluate returns was applied by Gibson and Dekkers (2008) because returns become increasingly less certain and valuable the further into the future they occur. Additionally, implementation of a GBP leads to a substantial reduction of generation interval due to the elimination of traditional PT schemes. As a novel idea of practical relevance, the modeling of the GBP in the current study accounted for a particular proportion of farmers waiting for daughter records of genotyped young bulls (YB-D), whereas only the more progressive farmers used young bulls (YB) based on their genomic indices as early as possible. These essential differences between the CPTP and the GBP are summarized in Table 4.

Two traits with moderate $\left(h^{2}=0.30\right.$; production $)$ and low heritability $\left(\mathrm{h}^{2}=0.05\right.$; functional trait) and an antagonistic relationship among each other $\left(\mathrm{r}_{\mathrm{g}}=-0.20\right.$; $\left.\mathrm{r}_{\mathrm{p}}=-0.10\right)$ were considered in the breeding goal with equal economic weights per genetic standard deviation. The distinction between the CPTP and GBP was achieved through modeling of information sources for selection index calculations. The GBP implied genomic indices (i.e., estimated genomic aggregate breeding values) in the base scenario with an accuracy (= square root of reliability) of 0.70 for all genotyped animals [i.e., YB, YB-D, and herdbook cows (HC) used as bull

Table 3. Important population parameters, biological and technical coefficients, and cost parameters for modeling the conventional progeny testing program (CPTP) in one region of Germany for Holstein dairy cattle

\begin{tabular}{lc}
\hline Input parameter & Numbers or costs \\
\hline Population parameter & 100,000 \\
Population size & 0.65 \\
Proportion of registered cows & 1.00 \\
Proportion of AI & 0.25 \\
Test capacity (i.e., proportion of recorded cows mated with test bulls) & 50 \\
Young bulls tested per year & 5 \\
Proven bulls selected per year, out of these & 10 \\
Inseminations per daughter lactation record & \\
Biological and technical coefficient & 1.15 \\
Average calving interval (in yr) & 2.40 \\
Inseminations per pregnancy & 0.15 \\
Proportion of losses during raising (female) & 3.00 \\
Use of proven bulls (yr) & 2.50 \\
Use of bull dams (yr) & 4.66 \\
Mean generation interval (yr) & \\
Cost parameter (Euro) & 31 \\
Milk recording costs per cow & 100 \\
Inspection of bull dam per selected bull dam & 5,000 \\
Keeping test bull on station per year (fixed and variable costs) & 1.5 \\
Herdbook registration per cow & 0.06 \\
Interest rate return & 0.04 \\
Interest rate costs & 15 \\
Investment period (yr) &
\end{tabular}


dams]. For those HC only used as cow dams and for all unregistered cows in the production unit, no genomic information in the index was assumed. Information sources used for these selection groups were own performances and performances of the dam leading to a correlation between aggregate genotype and index of 0.40. The CPTP (100 daughter records for production; 50 daughter records for the functional trait) used all available relatives' information for breeding value estimation as done in BLUP and, therefore, the accuracies of the indices (i.e., the correlation between aggregate genotype and index) were $0.81,0.55,0.61$, and 0.40 for $\mathrm{PB}, \mathrm{YB}, \mathrm{HC}$, and production cows, respectively.

The main criteria to evaluate the various breeding programs was the discounted profit (DP) calculated as the difference between the discounted returns (DR) and the discounted costs (DC) per cow. Discounted returns are calculated as the monetary value of the genetic superiority (= annual monetary genetic gain) expressed by improved animals in the whole population over the time of investment (15 yr).

\section{RESULTS AND DISCUSSION}

\section{Comparison of Conventional Progeny Testing and Genomic Breeding Programs}

Figure 3 shows the comparison of evaluation criteria for the CPTP and 4 scenarios of the GBP. In the scenarios GBP-YB and GBP-YB-D, 5 sires with highest genomic indices were selected out of 50 candidates as explained in Figure 2. Scenario GBP-YB-D implies an extension of the mean generation interval by $0.637 \mathrm{yr}$ because $50 \%$ of all inseminations were done by YB-D. The scenarios GBP-YB + SNP and GBP-YB-D + SNP considered the aspect of genotyping the initial sample size of 2,500 bulls. The effects of cost-saving due to the elimination of PT and of additional annual monetary genetic gain due to the reduction of generation intervals in GBP are greater compared with additional costs caused by genotyping of animals. Hence, a distinct economic advantage in DP was found for all scenarios of GBP in the range of factor 1.36 to 2.59. Model calculations for the Canadian Holstein population showed a doubling of selection response per year and a reduction of breeding costs by $92 \%$ when comparing GS with the traditional selection scheme (Schaeffer, 2006). In this study the most progressive scenario GBP-YB reduced costs by $22.4 \%$ only compared with CPTP because we assumed no changes in performance testing (e.g., no changes in the current milk recording scheme that is applied for all registered cows). Hence, the reduction of costs in the GPB is solely due to the elimination of keeping waiting bulls on station, but fixed costs for the breeding program were identical.

A stringent GBP as discussed by Schaeffer (2006) does not account for the proportion of farmers still waiting for daughter records of $\mathrm{YB}$, for production as well as for functional or type traits. From the practical point of view, some kind of YB-D as modeled in the present study seems to be the most realistic approach. Especially for Holstein dairy cattle breeding programs in Germany, information from visual inspections of progeny groups plays an important role when selecting cow sires for AI, and alternative testing schemes to improve the logistics of type trait classification were recently suggested by Swalve and König (2007). But even for the GBP-scenario where $50 \%$ of the inseminations are done by young bulls with daughter records and estimation of SNP effects in the initial sample (i.e., GBP-YB-D + SNP), DR and DP are increased by factors 1.17 and 1.36 , respectively, compared with the CPTP (Figure 3). Also, Schaeffer (2006) suggested setting up a system of cooperator herds within a GBP, but his intention was to simplify bull dam selection. The possibility to account for YB-D when modeling a GBP was not considered in previous studies, but should be carefully evaluated when discussing advantages of genomic selection. Results of a detailed variation of the percentage of inseminations done with YB-D in a GBP

Table 4. Essential differences in biological and technical coefficients and breeding costs when comparing conventional progeny testing program (CPTP) and genomic breeding program (GBP)

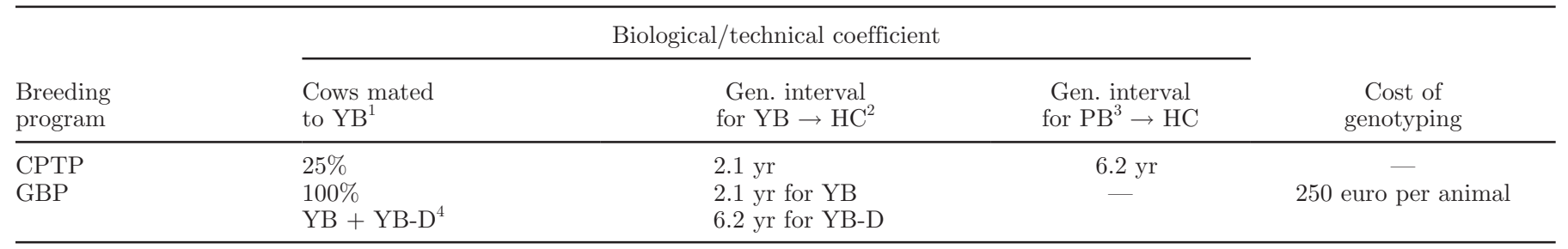

\footnotetext{
${ }^{1} \mathrm{YB}=$ young bulls used as cow sires.

${ }^{2} \mathrm{HC}=$ registered herdbook cows.

${ }^{3} \mathrm{~PB}=$ proven bulls.

${ }^{4} \mathrm{YB}-\mathrm{D}=$ genotyped young bulls with daughter records used as cow sires.
} 


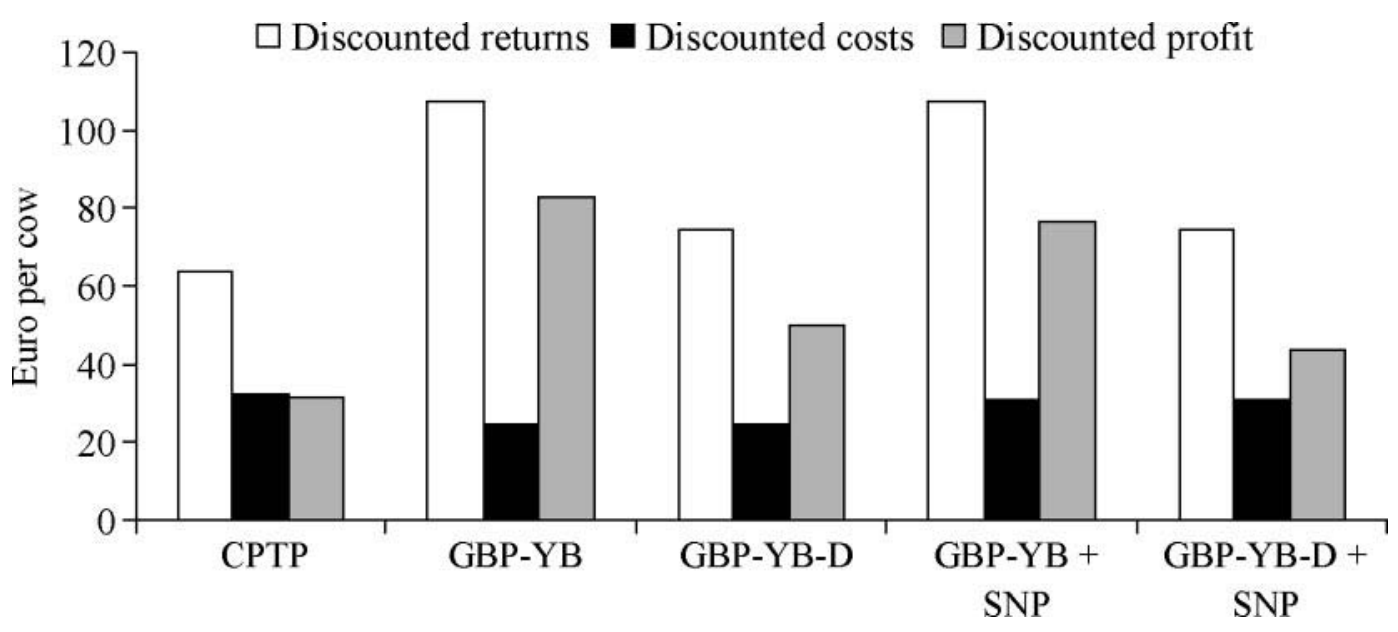

Figure 3. Discounted returns, costs, and profit for a conventional progeny testing program (CPTP) and 4 different scenarios of a genomic breeding program (GBP-YB $=100 \%$ of inseminations are done by genotyped young bulls without records; GBP-YB-D $=50 \%$ of inseminations are done by young bulls with daughter records; SNP = estimation of SNP effects in the initial sample size of 2,500 bulls).

are shown in Figure 4. Discounted returns and DP decreased linearly with increasing inseminations done by YB with daughter records (YB-D), due to the reduction of generation interval on the cow sire path. Other criteria influencing annual genetic gain (i.e., accuracies of genomic indices or selection intensities) are independent of the variation of YB-D in a GBP. Discounted costs were constant over the observed range because in all scenarios, $50 \mathrm{YB}$ were genotyped and 5 genotyped bulls were used as cow sires. When referring to the results presented in Figure 3 and Figure 4, a GBP only generates more DP compared with a CPTP if at least $20 \%$ of the inseminations were done with genotyped YB without daughter records.
One major obstacle in practical dairy cattle breeding programs is related to stagnating or even decreasing genetic trends for functional traits (König et al., 2007). Expectations for improvements were based on the incorporation of molecular genetic information in BLUP or selection index procedures, especially for low heritable traits (e.g., Lande and Thompson, 1990). The modeling in our study was to infer the change of the annual monetary genetic gain (AMGG) in both production and functionality when comparing the CPTP and GBP for different accuracies of genomic indices (0.70, $0.80,0.90$, and 0.99). As concluded in Figure 3 when evaluating DP over the investment period, AMGG was higher for the GBP and increased with increasing ac-

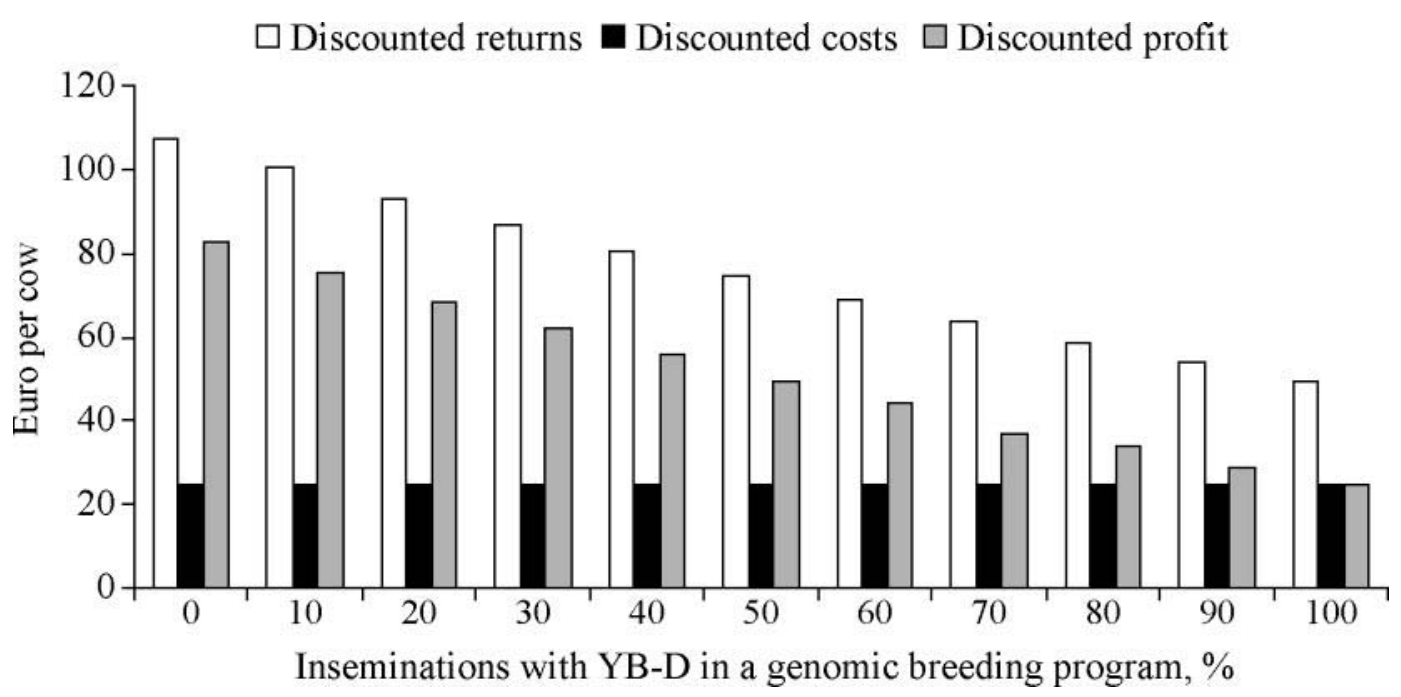

Figure 4. Discounted returns, costs, and profit for a genomic breeding program (GBP-YB) when different percentages of cows in the population are inseminated with genotyped young bulls having daughter records (YB-D). 


\section{$\square$ Production $\square$ Functionality Annual monetary genetic gain}

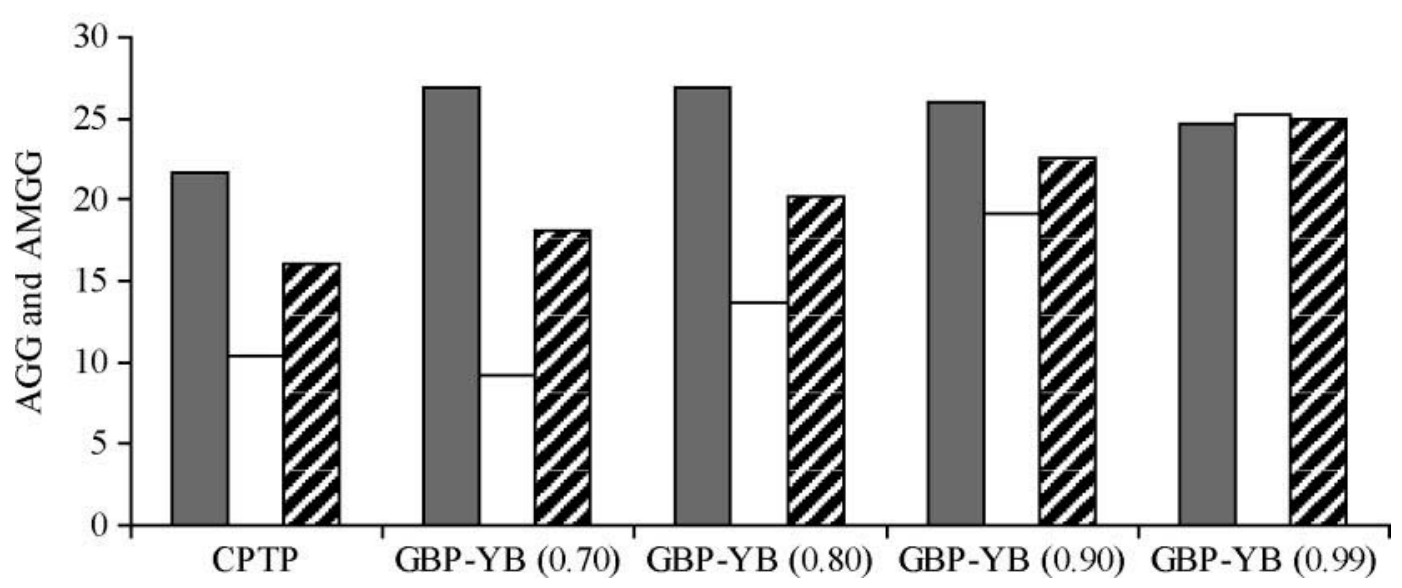

Figure 5. Annual genetic gain in production and functionality (AGG in genetic SD $\times 100$ ) and annual monetary genetic gain (AMGG) for a conventional progeny testing program (CPTP) and for a genomic breeding program (GBP-YB) when varying the accuracy of genomic indices $(100 \%$ of inseminations are done by genotyped young bulls without daughter records, no estimation of SNP effects in the initial sample size of 2,500 bulls), base scenario $=$ GBP-YB (0.70).

curacies of genomic indices (Figure 5). For an accuracy of 0.70 , AMGG is mostly due to the annual genetic gain (AGG) in production, whereas for extremely high accuracies of 0.90 or 0.99 , AGG for both components became similar. This implies a substantial increase of the AGG in the low heritable trait (functionality) and a minor decrease of the moderate heritable trait (production). Even higher success of GBP can be expected due to more optimistic assumptions when modeling the practical activities in the breeding program. In the current scenario, 100 genomic selected bull dams out of 200 preselected potential bull dams (Figure 2) are used to produce $50 \mathrm{YB}$. This implies a relatively high replacement rate of 0.5 at this stage of selection, being higher than the assumed replacement rate of 0.01 in the CPTP. Selection intensity for bull dams in a CPTP is traditionally high (König et al., 2007). Thus, changes of replacement rate of bull dams in large Holstein dairy cattle populations have no substantial impact on the AGG. In contrast, this parameter has a substantial impact in a GBP. Therefore, the AGG of the functional trait is lower in the GBP-YB (0.70) when compared with the CPTP (Figure 5). Preselection and genotyping of 1,000 potential bull dams would decrease the replacement rate to 0.1 . For this scenario and accuracies of genomic indices of 0.70, AGG (expressed in genetic SD multiplied by 100) in the production and functional trait was 32.89 and 11.35 , respectively. Hence, a distinct advantage in genetic gain over the CPTP was observed for both production and functionality. It has to be taken into account that a higher proportion of preselected cows for genotyping will increase the costs. Discounted costs increased from 24.80 to 26.80 euro per cow when increasing the number of preselected potential bull dams from 200 to 1,000 .

There would also be the possibility for breeding organizations to vary the selection intensity on the bull dam path when selecting bull dams according to genomic index. However, one constraint in the practical breeding program in our study was to generate $50 \mathrm{YB}$. Because embryo transfer or ovum pick up was not considered, the final selection of 100 bull dams has to be seen as a fixed number allowing only marginal variation.

\section{Various Scenarios of Genomic Breeding Programs}

The GBP-YB as explained in Figure 2 (but without estimation of SNP effects in the initial sample size) served as a base scenario. Alternatives focused on a) changes in selection intensity of cow sires in the final selection step $(5,6,10,15,20$, and 25 selected cow sires), b) changes in accuracies of genomic indices (0.4, $0.5,0.6,0.7,0.8,0.9,0.99)$, and c) changes in costs of genotyping $(50,150,200,250,300$, and 500 euro per genotyped animal).

A greater number of selected cow sires out of the pool of 50 genotyped YB in the final step of selection led to a decrease in selection intensity, lower DR and DP, and marginally higher DC due to keeping and feeding more bulls (Figure 6). When comparing the base scenario (selection of 5 cow sires, replacement rate $=$ 0.1 ) to 25 selected cow sires in the last selection step (replacement rate $=0.5$ ), DR and DP were decreased by factors 2.24 and 3.73 , respectively. This is due to the substantially lower annual genetic gain in both single traits (i.e., 0.15 genetic SD versus 0.27 genetic SD for 


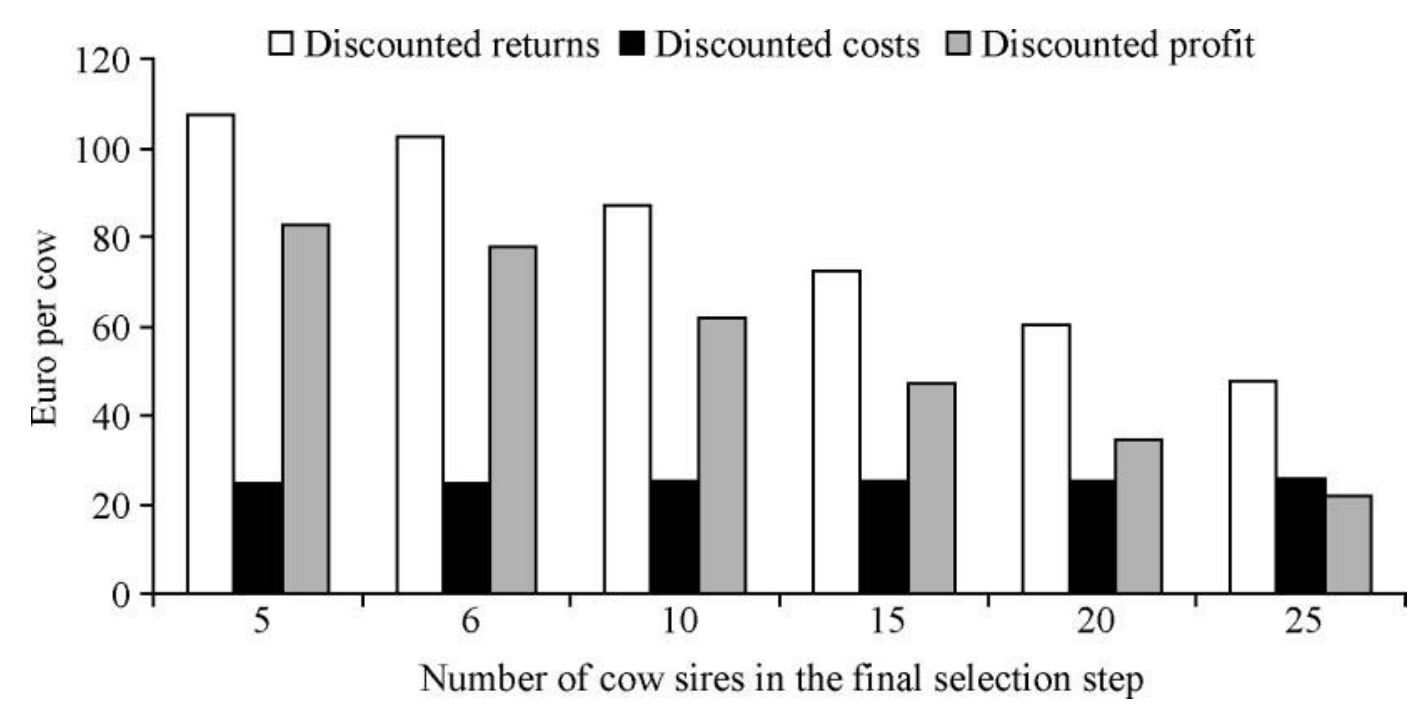

Figure 6. Discounted returns, costs, and profit for a genomic breeding program (GBP-YB) when varying the number of cow sires selected in the final selection step ( $100 \%$ of inseminations are done by genotyped young bulls without daughter records, no estimation of SNP effects in the initial sample size of 2,500 bulls), base scenario $=5$ cow sires.

the production trait, and 0.05 genetic SD versus 0.09 genetic SD for the functional trait). However, the use of only 5 sires (replacement rate of 0.1 ) for AI within a population of 100,000 cows can be a critical point when focusing on the aspect of inbreeding. As clearly pointed out by König and Simianer (2006), long-term control of inbreeding in a dairy population requires consideration of genetic relationships between young bulls entering AI breeding programs. Hence, for the optimal number of selected cow sires, aspects of inbreeding and relationships among selected candidates have to be considered. Schaeffer (2006) assumed additional potential from molecular data and suggested computing a heterozygosity index using the SNP genotypes. The implementation of such mating programs to maximize the heterozygosity index in the next generation, or even the inclusion of these tools in software packages for the evaluation of dairy cattle breeding programs, can provide more detailed information related to the optimal number of selected cow sires.

Discounted profit can be increased by approximately $50 \%$ if accuracy of genomic indices increases from 0.70 (base scenario) up to 0.99 (Figure 7). Hence, the development of accurate methods to compute reliable GEBV and therefore, genomic indices will be critical to the success of GBP, but different procedures lead to substantially different results (e.g., Meuwissen et al., 2001). When applying least squares analyses, BLUP, and Bayesian methods in their study, correlations between estimated and true breeding values were 0.32 , 0.73 , and 0.84 , respectively. More recent studies [e.g., Woolaston et al. (2007)] reported accuracies higher than 0.90 when applying principal component analysis, and even up to 0.98 for traits with heritabilities of 0.7 . Assuming a correlation between estimated and true aggregate breeding value of 0.99 , a GBP would increase DP by factor 3.95 compared with the CPTP (Figure 3 and Figure 7). Accuracies of about 0.4 in a GBP lead to similar results as in a CPTP when evaluating DP, but it is questionable if farmers select YB for inseminations if accuracies drop below 0.7 (König, 2004). Future research also has to identify whether same or different accuracies can be assumed for lowly and highly heritable traits. However, the range of accuracies in the study by Woolaston was quite small (i.e., from 0.98 to 0.78 when the heritability was 0.7 and 0.1 , respectively). Accuracies of GEBV and genomic indices will strongly depend on the quality of "conventional" EBV estimated with BLUP animal models (Swalve and König, 2007). This implies a need for ongoing collection of accurate phenotypic performances. Even in a GBP, breeding organizations have to maintain recording schemes for all essential traits, which was also considered as a cost component in the present study. As recently shown by Habier et al. (2007), it is also imperative to estimate the contribution from linkage disequilibrium to the accuracy of GEBV. As proven in their simulation study, decline of accuracies of GEBV in further generations was due to the decay of linkage disequilibrium raising the question of the optimal interval for reestimating of SNP effects.

Costs of genotyping were negligible when focusing on DC and DR per cow in the whole population for a given investment period as done in the present study. 


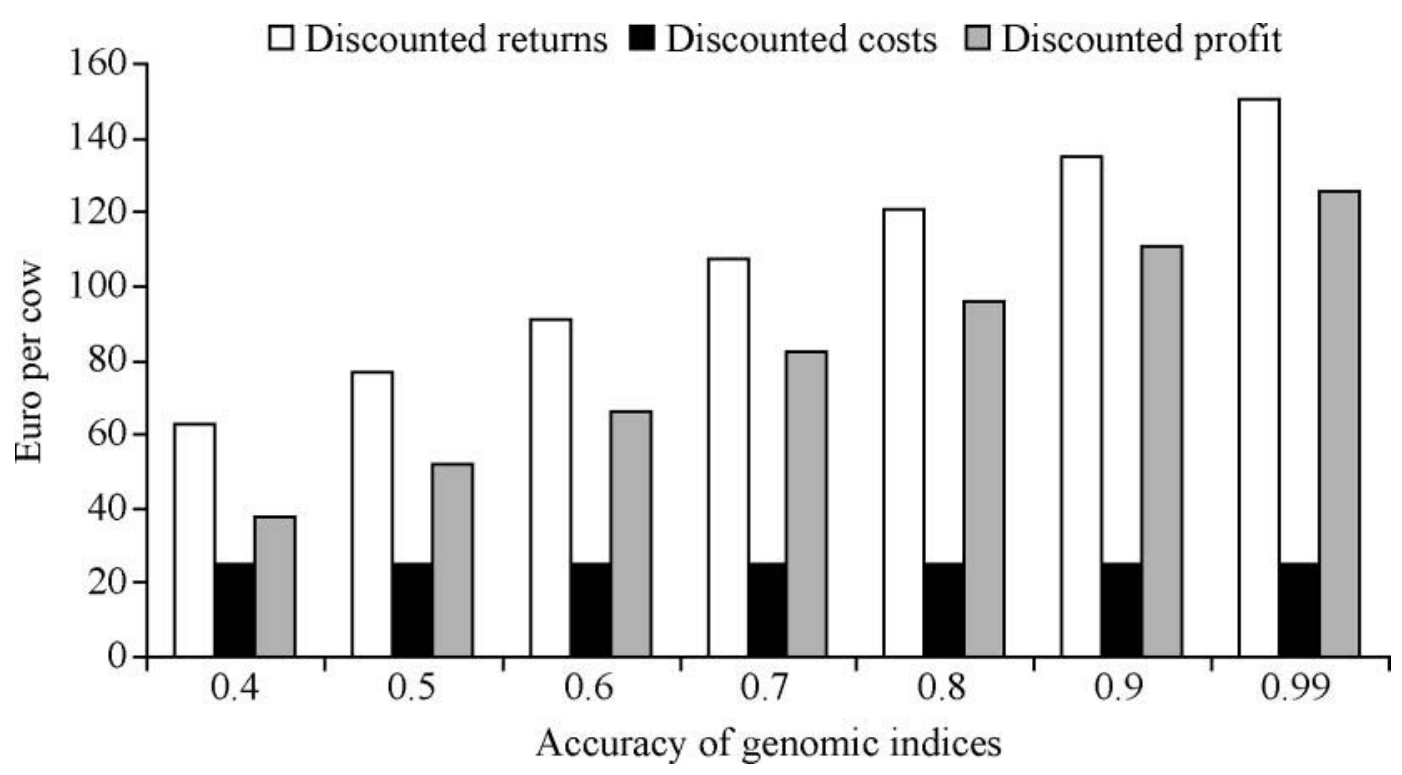

Figure 7. Discounted returns, costs, and profit for a genomic breeding program (GBP-YB) when varying the accuracy of genomic indices (100\% of inseminations are done by genotyped young bulls without daughter records, no estimation of SNP effects in the initial sample size of 2,500 bulls), base scenario $=0.7$.

Discounted costs only varied from 24.30 to 25.43 euro, when costs for genotyping increased from 50 to 500 euro per animal. An increase of costs for genotyping by 50 euro was associated with an increase in DC by 0.13 euro. Within a population of 100,000 cows, cost components such as herdbook registration, milk recording, or costs for keeping bulls are much higher than costs of genotyping $50 \mathrm{YB}$ and 200 potential bull dams. Hence, costs of genotyping had a very small impact when evaluating DP. Of more relevance is the question of intervals for recalculating SNP effects because at least 2,500 sires and sons were suggested in the studies by Meuwissen et al. (2001) and Schaeffer (2006). It can be assumed that the costs of genotyping per animal drop below 250 euro in the near future because high-density panels for genotyping 50,000 SNP are now commercially available and their costs are likely to decrease over time. Costs for genotyping have no impact on DR showing a constant value of 107.4 euro for all scenarios.

Results from the present study are strictly limited for a closed breeding program without importation of genetic improvement. Especially for Holstein dairy cattle, and due to the easy implementation of artificial insemination, selection schemes worldwide are characterized by the widespread use of the same genetically superior sires (Miglior, 2000). Gene flow of foreign sires from outside breeding programs was not modeled in this study, but would have substantial impact on the genetic gain in the whole population given that there is a large difference in genetic merit of domestic and international sires. Minor differences in genetic levels were found when comparing EBV of bulls or EBV of cows from different breeding organizations or even across county borders (König et al., 2007), mainly due to similar breeding strategies and close genetic relationships (König and Simianer, 2006). Hence, only minor modifications of main results are to be expected when extending the present study to an additional gene flow coming from foreign sires or even foreign bull dams. Additionally, the impact of variables such as costs for genotyping or accuracies of genomic indices on DP can be more clearly distinguished in a closed breeding program which is relatively free from outer noise.

\section{CONCLUSIONS}

Economic efficiency and increased annual genetic gain in dairy cattle breeding programs are possible due to the replacement of PT with genome-wide selection. Benefits arise largely from a substantial reduction in generation interval. Calculations for various breeding scenarios for the German Holstein population showed potential for more than a doubling of discounted profit when compared with the traditional PT selection scheme. Increasing the accuracy of genomic indices and increasing the selection intensity of cow sires had considerably more impact on the economic efficiency of GBP compared with the costs of genotyping animals. However, it will be interesting to see whether farmers accept genomic indices of YB without having knowledge of daughter records. This will be the crucial point for the practical implementation and ultimate success of 
GBP, and it has always been an underestimated effect when promising additional annual genetic gain through the application of new biotechnologies.

\section{REFERENCES}

Dekkers, J. C. M. 2007. Prediction of response to marker-assisted and genomic selection using selection index theory. J. Anim. Breed. Genet. 124:331-341.

Falconer, D. S., and T. C. F. Mackay. 1996. Introduction to Quantitative Genetics. Longman, Harlow, UK.

Gibson, J. P., and J. C. M. Dekkers. 2008. Design and economics of animal breeding strategies. http://www.une.edu.au/tigb/Gibsonbook/Chapter8.pdf Accessed Jun. 7, 2008.

Habier, D., R. L. Fernando, and J. C. M. Dekkers. 2007. The impact of genetic relationship information on genome-assisted breeding values. Genetics 177:2389-2397.

Henderson, C. R. 1964. Selecting the young sire to sample in artificial insemination. J. Dairy Sci. 47:439-441.

Hill, W. G. 1974. Prediction and evaluation of response to selection with overlapping generations. Anim. Prod. 18:117-139.

Kolbehdari, D., L. R. Schaeffer, and J. A. B. Robinson. 2007. Estimation of genome-wide haplotype effects in half-sib designs. J. Anim. Breed. Genet. 124:356-361.

König, S. 2004. Züchtungsfragen: Nutzungsdauer und Fruchtbarkeit im Focus. Pages 97-119 in Trendreport Spitzenbetriebe. DLGVerlag, Frankfurt/Main, Germany.

König, S., S. Lessner, and H. Simianer. 2007. Application of controlling instruments for improvements in cow sire selection. J. Dairy Sci. 90:1967-1980.

König, S., and H. Simianer. 2006. Approaches to the management of inbreeding and relationship in the German Holstein dairy cattle population. Livest. Sci. 103:40-53.

Lande, R., and R. Thompson. 1990. The efficiency of marker assisted selection in dairy cattle breeding schemes. Genetics 124:743753 .
Leisen, M. 1999. Erfolgsfaktoren innovativer Zuchtprogramme. Seminar. ADR Bonn 12

Long, N., D. Gianola, G. J. M. Rosa, K. A. Weigel, and S. Avendano. 2007. Machine learning classification procedure for selecting SNPs in genomic selection: Application to early mortality in broilers. J. Anim. Breed. Genet. 124:377-389.

Mackinnon, M. J., and M. George. 1998. Marker-assisted selection of young dairy bulls prior to progeny testing. Livest. Prod. Sci. $54: 229-250$.

Meuwissen, T. H. E., B. Hayes, and M. E. Goddard. 2001. Prediction of total genetic value using genome-wide dense marker maps. Genetics 157:1819-1829.

Miglior, F. 2000. Impact of inbreeding-Managing a declining Holstein pool. Pages 108-113 in Proc. 10th World Holstein Friesian Conf., Sydney, Australia.

Nicholas, F. W., and C. Smith. 1983. Increased rates of genetic change in dairy cattle by embryo transfer and splitting. Anim. Prod. Sci. 36:341-353.

Nitter, G., H. Bartenschlager, K. Karras, E. Niebel, and H. U. Graser. ZPLAN: A PC program to optimize livestock selection programs. User's Guide for ZPLAN, Version 2007, University Hohenheim and University of New England, Armidale, Australia.

Rendel, J. M., and A. Robertson. 1950. The use of progeny testing with artificial insemination in dairy cattle. J. Genet. 50:21-31.

Schaeffer, L. R. 2006. Strategy for applying genome-wide selection in dairy cattle. J. Anim. Breed. Genet. 123:218-223.

Swalve, H. H., and S. König. 2007. Test herds in dairy cattle breeding programmes: 1st communication: General considerations. Züchtungskunde 79:449-462.

Willam, A., C. Egger-Danner, J. Sölkner, and E. Gierzinger. 2002. Optimization of progeny testing schemes when functional traits play an important role in the total merit index. Livest. Prod. Sci. $77: 217-225$.

Woolaston, A. F., B. Tier, and R. D. Murrison. 2007. Principal components regression of SNP data to predict genetic merit. Page 80 in Proc. Workshop on QTL and Marker Assisted Selection 2223 March 2007. Toulouse, France. 